\title{
COHOMOLOGY OF CERTAIN STEINBERG GROUPS
}

\section{BY DAVID HERTZIG}

Communicated by D. Buchsbaum, March 25, 1968

In [3] Steinberg considers the relations satisfied by generators of the Chevalley groups and defines certain abstract groups $\Delta$ and $\Gamma$ via presentation. Let $\Sigma$ be a root system of a simple complex Lie algebra $\mathcal{B S}_{C}$ and let $K$ be a field of characteristic $p \geqq 0$. We consider a set of generators $x_{r}(t)(r \in \Sigma, t \in K)$ and the relations

$$
\begin{aligned}
& \text { (A) } x_{r}(t) x_{r}(u)=x_{r}(t+u) \quad(r \in \Sigma ; t, u \in K), \\
& \text { (B) } x_{r}(t) x_{s}(u) x_{r}(t)^{-1}=x_{s}(u) \prod x_{i r+j s}\left(C_{i j ; r s} t^{i} u^{j}\right) \\
& \quad(r, s \in \Sigma, r+s \neq 0 ; t, u \in K) .
\end{aligned}
$$

The product in (B) is over all integers $i, j \geqq 1$ for which $i r+j s \in \Sigma$, taken in lexicographic order. The $C_{i j ; r s}$ are certain integers depending only on the structure of $\mathbb{S}_{C}$ (cf. [1]). Steinberg defines $w_{r}(t)$ $=x_{r}(t) x_{-r}\left(-t^{-1}\right) x_{r}(t)$ and $h_{r}(t)=w_{r}(t) w_{r}(-1) \quad\left(r \in \Sigma ; t \in K^{*}\right)$ and considers also the relations

(B') $w_{r}(t) x_{r}(u) w_{r}\left(t^{-1}\right)=x_{-r}\left(-t^{-2} u\right) \quad\left(r \in \Sigma ; t \in K^{*}, u \in K\right)$,

(C) $h_{r}(t) h_{r}(u)=h_{r}(t u) \quad\left(r \in \Sigma ; t, u \in K^{*}\right)$.

The Steinberg group $\Delta$ is the abstract group generated by the symbols $x_{r}(t)(r \in \Sigma ; t \in K)$ subject to the relations (A) and (B) if the rank of $\Sigma$ is $>1$, to the relations (A) and $\left(B^{\prime}\right)$ if the rank of $\Sigma=1$. The Steinberg group $\Gamma$ is the abstract group with the same generators as $\Delta$ subject to the relations of $\Delta$ and in addition subject to the relations (C).

In [1] Chevalley constructs a corresponding Lie algebra $\$$ over the field $K$ and there is a natural action of the Steinberg groups on $\$ 5$. One is then led to consideration of the cohomology $H^{1}(\Delta, \Theta)$ and $H^{1}(\Gamma$, (s).

The author has developed a technique for computation of such cohomology (cf. [2]). This is applied successfully to obtain the following results. Proofs will appear elsewhere.

We denote $D(K)$ the module of derivations of $K$. In the case of characteristic $p=2$ we denote $\mathcal{L}(K)$ the $K^{2}$-linear transformations $L$ of $K$ such that $L(1)=0$. Since $p=2, D(K) \subset \mathscr{L}(K)$, but in general $\mathscr{D}(K) \neq \mathscr{L}(K)$.

THEOREM 1. $H^{1}(\Delta,(\Im))=H^{1}(\Gamma, \Im) \cong D(K)$ in the following cases:

(i) type $A_{1}, p \neq 2$ and $K \neq F_{5}$; 
(ii) type $A_{n}(n \geqq 2), p \nmid n+1$;

(iii) type $D_{n}(n \geqq 4), p \neq 2$;

(iv) type $E_{6}, p \neq 3$;

(v) type $E_{7}, p \neq 2$;

(vi) type $E_{8}$;

(vii) type $F_{4}$.

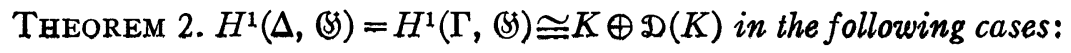

(i) type $A_{n}(n \geqq 2), p \mid n+1$;

(ii) type $D_{n}(n \geqq 4, n$ odd $), p=2$;

(iii) type $E_{6}, p=3$;

(iv) type $E_{7}, p=2$.

Theorem 3. $H^{1}(\Delta, \mathbb{S})=H^{1}(\Gamma, \mathfrak{S}) \cong K \oplus K \oplus \mathbb{D}(K)$ in the case: type $D_{n}(n \geqq 4, n$ even $), p=2$.

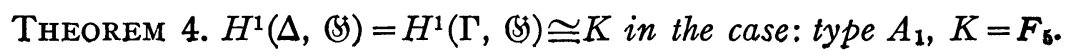

THEOREM 5. $H^{1}(\Delta, \mathfrak{S}) \cong K \oplus \mathscr{L}(K), H^{1}(\Gamma, \mathfrak{B}) \cong K \oplus \mathbb{D}(K)$ in the case: type $A_{1}, p=2$.

\section{REFERENCES}

1. C. Chevalley, Sur certains groupes simples, Tôhoku Math. J. (2) 7 (1956), 14-66.

2. D. Hertzig, Cohomology of algebraic groups, J. Algebra 6 (1967), 317-334.

3. R. Steinberg, Génêrateurs, relations et revêtements de groupes algêbriques, Colloque Theory des Groupes Algebriques, Brussels Libraire Universitaire Louvain and Gauthier-Villars, Paris 1962, pp. 113-127.

Purdue University, Lafayette, Indiana 47907 